\title{
L'Homme
}

Revue française d'anthropologie

$191 \mid 2009$

Des femmes en noir...

\section{Le chant qui donne la vie}

Ou comment Molly Malone est devenue vivante. Un processus mythifiant au cœur de Dublin

A Life-Giving Song : How Molly Malone Came to Life. A Myth-Making Process in Dublin

\section{Charlotte Poulet}

\section{OpenEdition}

\section{Journals}

Édition électronique

URL : http://journals.openedition.org/lhomme/22232

DOI : 10.4000//homme.22232

ISSN : 1953-8103

Éditeur

Éditions de l'EHESS

Édition imprimée

Date de publication : 1 janvier 2009

Pagination : 175-200

ISSN : 0439-4216

Référence électronique

Charlotte Poulet, «Le chant qui donne la vie », L'Homme [En ligne], 191 | 2009, mis en ligne le 01 janvier 2011, consulté le 19 avril 2019. URL : http://journals.openedition.org//homme/22232 ; DOI : 10.4000/ Ihomme.22232

(c) École des hautes études en sciences sociales 


\title{
Le chant qui donne la vie Ou comment Molly Malone est devenue vivante. Un processus mythifiant au cœur de Dublin
}

\section{Charlotte Poulet}

\author{
Now her ghost wheels her barrow, \\ Through streets broad and narrow, \\ "Crying, Cockles and mussels, alive, alive oh!"
}

M

Olly Malone, héroïne de la chanson irlandaise Cockles and Mussels, connaît une gloire sans précédent. Cette Marianne irlandaise est, en effet, devenue l'emblème de Dublin - sa ville natale -, et, plus largement, de l'Irlande. Nombre de pubs dans le monde portent son nom comme une bannière aussi voyante qu'une enseigne de Guinness. Ce sont des milliers de touristes qui, chaque année, se pressent autour de sa statue érigée au cour de la ville. La chanson qui l'évoque n'en est pas pour autant délaissée, entonnée et scandée comme hymne officieux de la ville. Elle résonne dans les stades, chantée par les supporters de l'équipe de Dublin de football gaélique (GAA), tout autant que par ceux de l'équipe internationale de rugby. Fétiche également des musiciens irlandais, nous la retrouvons sur de nombreux enregistrements tels que ceux de The Pogues, The Dubliners, Sinead O'Connor, etc.

Mais qui est donc Molly Malone? Si l'on s'en tient au texte de la chanson, Molly Malone était une belle poissonnière déambulant dans les rues de Dublin, mais qui succomba, trop jeune, à une fièvre. Cela suffit-il à la graver dans les mémoires?

L'histoire de cette chanson et de ses tribulations dublinoises sera mieux à même de nous faire comprendre le "processus héroïsant " mis à l'œuvre ici. Le texte inerte, imprimé, ne peut en dire plus; c'est par le chant - le chanter - qu'il prend corps et vie, par les voix de tous et de chacun, par l'ensemble des interactions sous-jacentes à toute pratique et, en particulier, à celles qui se font jour à travers le chant. Il s'agit de rendre Molly Malone à son contexte d'énonciation, à cette Irlande où pas un soir ne se passe sans qu'un chant ne s'élève d'un pub. 
Cockles And Mussels est une chanson parmi bien d'autres au sein du répertoire traditionnel irlandais ${ }^{1}$. Ce répertoire est composé de ballads pour la plupart anonymes et non datées. Elles circulent oralement à l'occasion de réunions au pub entre amis ou bien en famille. Elles sont tout autant l'objet de l'intimité villageoise que du genre musical irlandais qui se diffuse et se produit désormais au-delà des frontières qui l'ont vu naître. Aux confins d'une pratique formelle et d'une pratique informelle, de l'intime et du public, elles font parties intégrantes de la vie quotidienne et, plus particulièrement, des pratiques sociales irlandaises.

En mobilisant l'approche anthropologique, il s'agit de comprendre comment Molly Malone est devenue vivante, c'est-à-dire par quels processus symboliques l'esprit d'une ville s'est incarné dans le personnage d'une chanson. Nous reviendrons sur les faits qui donnèrent peu à peu vie à Molly Malone grâce à l'ouvrage de Sean Murphy (1992) qui en retrace les temps forts. À ces données historiques et historiographiques sera superposée une réflexion sur la pratique du chant, se fondant sur l'ethnographie que $j$ 'ai pu en faire ${ }^{2}$, afin d'apporter une relecture des faits et une compréhension du processus en ouvre. À la manière dont Richard Bauman (1986) a analysé la littérature orale, c'est la relation entre l'événement de chanter, les événements chantés et le texte que je me propose d'explorer.

\section{Au commencement était le texte...}

\section{Les enjeux de l'origine}

Dans son livre The Mystery of Molly Malone, Sean Murphy (1992) relate les vicissitudes auxquelles donna lieu la circulation de cette chanson, à Dublin. Il nous révèle ainsi que des débats sont apparus lorsqu'une version publiée du texte ${ }^{3}$ fut mise au jour à la suite de recherches sur l'origine de cette chanson. Il résume les trois principaux sujets de discorde qui sont alors survenus.

Le premier problème posé par l'existence du texte fut la signature d'un auteur: James Yorkston. Cette attribution souleva l'opinion dublinoise; l'incompréhension fut générale : le texte des paroles ne pouvait décemment pas être antérieur à la version orale. La question fut réglée simplement et

1. J'appelle ici «répertoire traditionnel irlandais » les chansons qui figurent dans les publications se réclamant de ce nom comme par exemple celui-ci : 100 Irish Ballads, Dublin, Soodlum, 1981. Le texte traduit de Cockles And Mussels pourra être trouvé en annexe.

2. L'ethnographie de la pratique informelle du chant, au pub, a été l'objet de mon travail de DEA, en 2006 ; puis de ma première année de doctorat en 2007.

3. Texte publié à Londres en 1884 par Francis Brothers \& Day. 


\section{Cockles and Mussels}
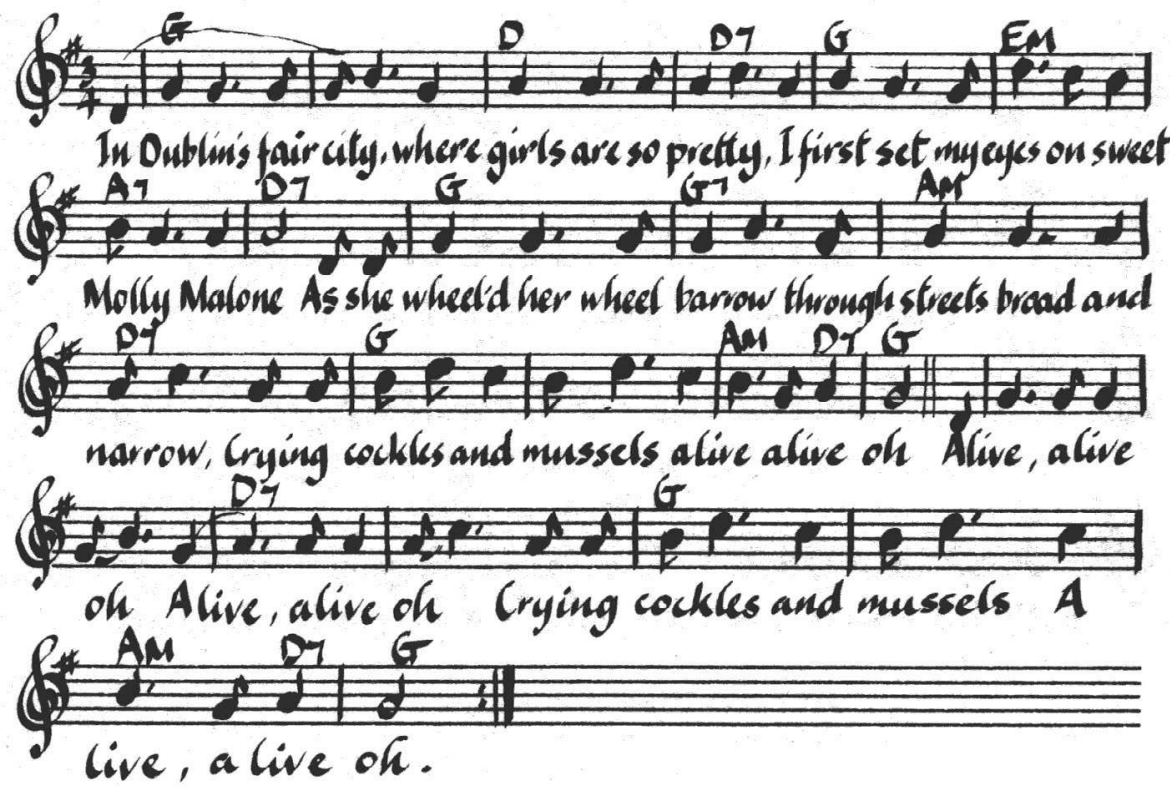

Transcription extraite de N. James Healy, Ballads from the Pubs of Ireland.

Popular Songs and Ballads. Cork, Ossian, 1996 [1965]: vol. I, 54.

sévèrement: James Yorkston ne pouvait qu'avoir effectué un plagia de la tradition orale, cherchant dès lors à s'approprier un symbole de la création collective, anonyme, traditionnelle. En deuxième lieu, l'attention se porta sur la date attribuée au texte: 1884. Or, une chanson traditionnelle ne devait absolument pas exhiber une date d'écriture, en tout cas pas aussi récente. Cockles And Mussels fut donc, à la suite de cette découverte, écartée de la catégorie "traditionnel» du fait de son manque d'ancienneté, et rangée parmi les chansons populaires modernes. Le troisième point sensible de cette affaire, selon Sean Murphy, est que James Yorkston était d'origine écossaise et, plus précisément, résidait à Edinburgh. Comment pouvait-on dès lors aduler une chanson écrite par un Écossais? Molly Malone serait donc écossaise et non irlandaise, et, pourtant, la chanson commence ainsi : "In Dublin's fair city, where the girls are so pretty". Selon le principe que toute localisation ne pouvait être induite que par le lieu même de la naissance des chansons, plusieurs hypothèses furent avancées afin de justifier les premières paroles de Cockles And Mussels, à commencer par celle-ci : peut-être Molly Malone n'était-elle finalement pas une 
personne concrète mais un prototype forgé par l'auteur, et peut-être même en avait-il trouvé l'inspiration lors d'un voyage à Dublin... Mais toutes ces questions ne furent jamais élucidées et ne le seront probablement jamais.

Notons toutefois que ces questions revêtent une dimension anthropologique si on évoque le problème que se sont posé nombre de chercheurs au sujet de la classification des artefacts à l'intérieur d'une tradition orale qui, par ailleurs, ne méconnaît pas l'écriture. Alors que l'International Folk Music Council ${ }^{4}$ proposait une définition (1954) de la musique traditionnelle assez vaste pour englober de nombreux objets en posant comme conditions de l'authenticité un processus de circulation orale et une continuité avec le passé, certains y perçurent l'idée de nécessité d'une création orale et d'une ancienneté immémoriale. C'est ainsi qu'en Irlande, James Yorkston ne fut pas le seul à être écarté du patrimoine musical. John Moulden (1997) relate un phénomène identique relatif au poète Hugh McWilliams. Toutefois, il démontre habilement, par une étude syntaxique et historique, que les versions orales partent toutes d'une même origine: la version écrite. Pour nous résumer sur ce point, nous pourrions dire que Cockles And Mussels, en éveillant les consciences puristes de la tradition, nous amène à penser à ce qu'une chanson doit être pour un groupe : elle doit lui être immanente dans le sens où elle ne saurait accepter qu'un seul individu en soit auteur; elle doit être non datée pour que la formule "on la chante depuis toujours» soit non seulement appropriée mais permise ; elle doit être enracinée localement pour qu'elle appartienne au groupe et lui propose une identification. Ainsi le propre d'un objet de la tradition serait-il l'anonymat, la localisation et l'atemporalité.

Il est important de rappeler que les controverses rapportées par Sean Murphy se sont situées dans la sphère universitaire dublinoise. Pendant ce temps, Cockles And Mussels continuait son chemin, chantée comme à l'accoutumée, au pub, par la population dublinoise qui ne doutait guère que cette chanson pût être autrement que traditionnelle; après tout, elle figure bien dans tous les répertoires se revendiquant comme tels. Nous pouvons donc observer que si on détache le texte de sa pratique, il n'est plus traditionnel au sens même où il avait été défini.

4. Cette définition a été proposée lors du congrès de Sao Paulo et publiée dans le Journal of the IFMC, 1955: «Folk music is the product of a musical tradition that has been evolved through the process of oral transmission. The factors that shape the tradition are : a) continuity which links the present with the past ; b) variation which springs from the individual or the group ; c) selection by the community, which determines the form or forms in which music survives" ("La musique populaire est le produit d'une tradition musicale évoluant au travers d'un processus de transmission orale. Les facteurs qui la constituent sont: a) la continuité reliant le passé au présent; b) les variations émergeant d'une pulsion créatrice individuelle ou de groupes; c) la sélection par la communauté qui détermine la forme ou les formes sous lesquelles la musique survit "; ma traduction). 
Avec le temps, les esprits se sont apaisés, mais les opinions restent tranchées, les uns défendant l'acte collectif de création, les autres demeurant soucieux de restituer les produits à leurs véritables auteurs. Sean Murphy remarque que chaque éditeur de répertoire de chansons, chaque artiste reprenant Cockles And Mussels, affirme ainsi sa position en apposant, ou non, le nom de James Yorkston à la partition. Et, en effet, après quelques recherches, nous trouvons parfois le texte de la chanson alors anonymisée mais sans intention mensongère: "The author of this famous Dublin song is unknown " 5 , et d'autres fois signée comme, par exemple, dans la bande-son originale du film Orange Mécanique de Stanley Kubrick.

Mais où est donc Molly Malone?

Les intellectuels et universitaires dublinois ne se résolurent pas à abandonner la partie et à laisser Cockles And Mussels sous la plume d'un Écossais (avec comme première motivation, celle rappelée ci-dessus : à savoir que c'est à Dublin qu'elle fut et était chantée depuis) ; ils cherchèrent alors un moyen autre que celui du texte pour démontrer que l'acte de chanter cette chanson hic et nunc était parfaitement légitime et relevait bien de l'esprit dublinois sinon irlandais. Le moyen le plus simple de prouver que Molly Malone appartenait bien à l'Irlande, et plus particulièrement à Dublin, n'était-il pas de la retrouver, elle... derrière les paroles du texte. Sean Murphy nous raconte cette quête effrénée.

C'est grâce à un individu, jusque-là inconnu des sphères universitaires ou artistiques, que l'idée que Molly Malone aurait réellement vécu à Dublin prit corps. Selon lui, elle serait enterrée au cimetière de St John près de Fishamble Street. Cette rumeur s'amplifia lorsque cette information fut rapportée et étayée dans un ouvrage sérieux ${ }^{6}$, venant ainsi à l'appui de considérations, propos et opinions qui s'opposaient à la destruction du site par une société de travaux publics. Afin de rendre crédible l'argument, une date de naissance fut donnée à Molly Malone : 1734. Mais, en dépit de ce motif bien trouvé, et apparemment fondé, la tentative de sauvegarde du site échoua, et la Dublin Corporation construisit la route prévue. Toutefois, l'idée que la vie de Molly Malone pouvait être réellement retracée s'imposa. C'est ainsi que lors d'une visite d'un académicien américain, l'hypothèse fut émise que Molly Malone était forcément morte pendant l'épidémie de fièvre typhoïde, sévissant alors, et qui se transmettait par la consommation de coques et de moules (cockles and mussels)

5. 100 Irish Ballads, Dublin, Soodlum, 1981: 29.

6. Cf. Bradley (1984). 
contaminées provenant de la baie de Dublin. Tous les éléments de l'histoire coïncidaient, tout devenait désormais évident. La tenue du Dublin Millenium en $1988^{7}$ acheva d'inscrire et de répandre cette légende naissante dans les esprits. Plus aucun doute n'était permis : la légende se transformait bel et bien en vérité historique. Et les historiens réticents furent rapidement congédiés. Le 22 janvier 1988, lors d'une conférence de presse à la St Andrew's Church, à l'occasion de la promotion du spectacle Dublin's Fair City, il fut annoncé, officiellement et solennellement, que les actes de baptême, de décès et d'enterrement de Molly Malone avaient été découverts dans les registres de la St John's Church ${ }^{8}$. Une personne répondant au nom de Mary Malone avait été baptisée le 27 juillet 1663 et enterrée le 13 juin 1699. La St John's Church et son cimetière étaient situés près de Fishamble Street (tous deux furent démolis au siècle dernier) et appartenaient à la confession anglicane, The Church of Ireland. Comme le remarque à juste titre Sean Murphy, "Molly » est une variante du prénom "Mary», mais aucune trace ne pouvait laisser supposer qu'une Mary, parmi toutes celles enregistrées, portait le surnom de Molly. En outre, The Church of Ireland compte bien plus d'une Mary Malone dans ses registres et peut-être même que The Roman Catholic (confession catholique romaine) en compte davantage. Toutefois, un baptême à la Church of Ireland semblait préférable pour Molly Malone, tout autant que semblait l'être ce XVII siècle qui l'aurait vu naître. Grâce à cette généalogie parfaite et historiquement reconstruite sinon avérée, Molly Malone pouvait désormais être une Dublinoise d'exception et devenir l'objet de vénération, si ce n'est même d'idolâtrie. En cette même année, la commission du Dublin Millenium se réunit avec à sa tête Matt McNulty, et la décision fut prise d'ériger une statue en l'honneur de cette poissonnière devenue célèbre, mais à l'existence de laquelle tous n'avaient pas voulu croire. La sculptrice, Jeanne Rynhart remporta le concours agrémenté d'un projet proposant des recherches sur le contexte historique de la vie de Molly Malone. Ces recherches eurent pour but surtout de réunir et d'unifier les rumeurs, et quelques éléments furent également ajoutés. De la sorte, Molly fut revêtue d'une robe du XVII e siècle qui la figurait comme une commerçante enrichie par des activités de prostitution. Les rues où elle exerçait furent par la suite identifiées comme étant Grafton Street et St Stephen Green; sa réputation ainsi établie, il fut déclaré qu’elle avait des clients, renommés pour leur débauche, au Trinity College. Une deuxième version réinterprétée

7. Ce millenium n'est pas en réalité celui de la fondation de la ville (841), mais de la prise de celle-ci par Maol Sechnaill II en 989.

8. Événement relaté dans le Irish Times du 23 janvier 1988. 
de Cockles And Mussels se répandit alors dans les pubs dublinois sous le titre: Sixpence For The Night. En 1989, la statue fut dressée au carrefour de Grafton Street et Sufolk Street, lieu où Molly fut censée exercer ses activités. Une seconde raison fut apportée à ce choix, ce croisement est aussi celui où se dresse la St Andrew Church, lieu de son baptême. Rappelons qu'elle avait été précédemment déclarée baptisée à la St John's Church, toutefois, cette déclaration avait été faite à la St Andrew Church. Cette confusion n'est, en réalité, rien d'autre qu'une mauvaise lecture de l'article de presse rendant compte de la conférence. La St Andrew Church ne fut construite qu'en 1665 et ses registres ont été détruits en 1922.

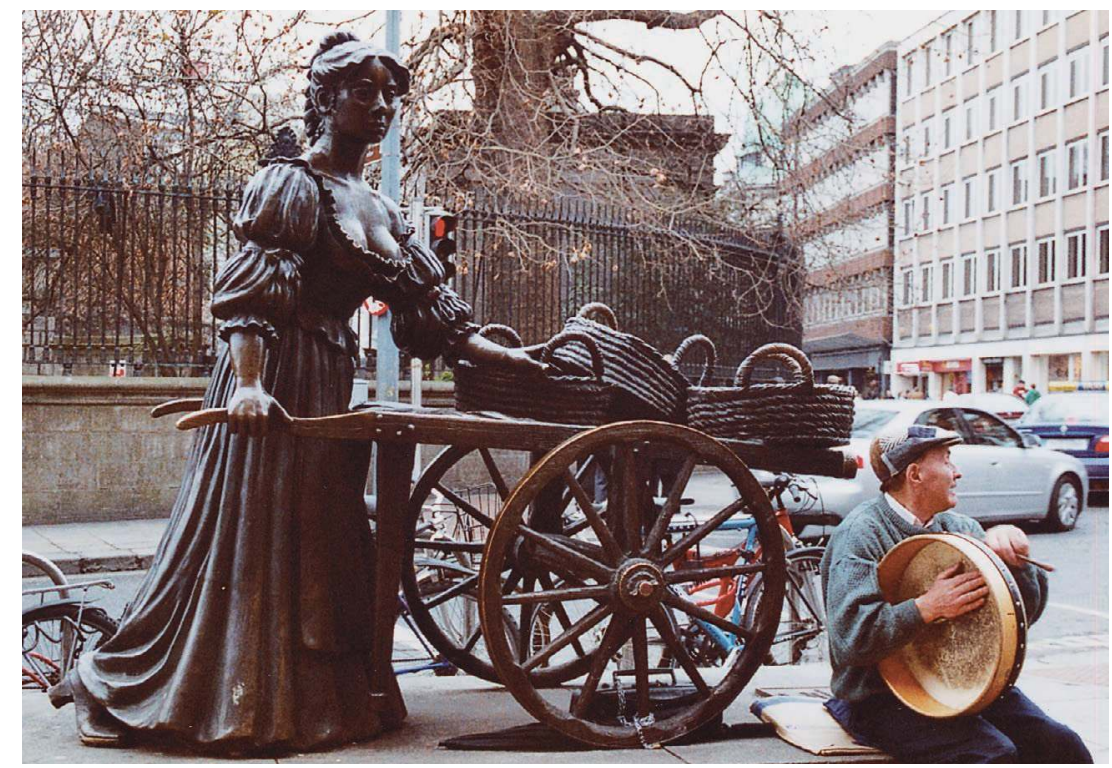

Statue de Molly Malone, érigée en 1989 au carrefour de Grafton Street et Sufolk Street, à Dublin (cl. Charlotte Poulet).

Dix ans après avoir redonné vie et visage à Molly Malone, l'idée d'une célébration annuelle prit forme. Le 13 du mois de juin 1999 (300ªnniversaire de sa mort) fut désigné comme l'International Molly Malone Day; dans le même temps la Molly Malone Summer School vit le jour. Et les rumeurs reprirent de plus belle... Le 7 juin 1999, des universitaires suggérèrent, lors d'une émission de radio, que Molly Malone était en réalité Peg Woffington, la maîtresse dublinoise de Charles II, les coques et les moules étant les symboles du sexe féminin. Ces rumeurs incessantes, recueillies par Sean Murphy, sont finalement le fruit d'une légende urbaine ayant pris racine lorsque les premiers débats sur Cockles And Mussels eurent lieu. Cette légende a donné vie, voire naissance, à Molly Malone, désormais symbole de l'héritage culturel de Dublin. 
Si Cockles And Mussels a pris une telle ampleur en une vingtaine d'années, c'est que - telle est du moins mon hypothèse - les spécificités des ballads irlandaises, tout autant que celles de l'acte de chanter, ont permis une telle mythification urbaine. C'est en explorant ces domaines que nous serons mieux à même de comprendre ce qui s'est passé, et quel processus a pu engendrer l'«existence historique» de Molly Malone. Ainsi, poser la question de comment les intellectuels dublinois ont pu rendre vivante Molly Malone, revient-il à s'interroger sur la pratique de ceux à qui elle appartient, de ceux qui la chantent.

\section{Chanter}

\section{Les ballads}

Le répertoire irlandais est essentiellement composé de ballads. Cette terminologie a été développée par les folkloristes anglophones; les équivalents français restent approximatifs (ils désignent plutôt les chansons des troubadours des XIV et $\mathrm{XV}^{\mathrm{e}}$ siècles), même si ce type de chansons n'est pas spécifique au domaine anglo-saxon. Les folkloristes, qui ont fait de cette notion l'objet de leurs études, ne s'accordent pas tous sur l'origine supposée de ces chants, oscillant entre expression individuelle et collective. Toutefois, un terrain d'entente fut trouvé lorsqu'il s'est agi de constater une indéniable dégradation du matériel par la reprise systématique des morceaux originaux. En effet, le propre de la ballad est la circulation orale reformulant indéfiniment le texte jusqu'à en perdre l'origine. Gordon Hall Gerould (1974) et Alan Lomax, par son œuvre, nous apportent une définition convergente du contenu de la ballad, quels que soient par ailleurs leurs points de désaccord. Une ballad est toujours un récit contenant une petite histoire se fondant sur la vie quotidienne, qu'elle soit romancée ou véridique. Le ton est impersonnel, c'est-à-dire que les éléments de l'histoire parlent d'eux-mêmes, et il n'y a pas d'émission de jugement, ni de morale concluante, mais plutôt une posture dramatique du narrateur amenant à une compréhension émotionnelle par les auditeurs. La ballad s'adapte donc à son contexte d'énonciation en insistant sur la situation dans le récit plutôt que sur la continuité de la narration ou sur la description des personnages. Nous pourrions alors dire, afin d'être plus précis, que les récits ainsi musiqués sont de l'ordre de l'anecdote. Richard Bauman (1999 : 54-60) décrit cette notion en ces termes: une histoire courte, un incident vrai avec des personnages réels, les événements et les personnages étant situés dans le temps et dans l'espace. Nous remarquons, en effet, au vu des répertoires publiés, que toutes les chansons se rapportent à des thèmes de la vie quotidienne (l'amour, la guerre, l'exil, la fête, etc.). 
Ces derniers sont toujours abordés par le narrateur-chanteur de manière subjective, et donc personnelle, par la restitution d'incidents ou d'événements qu'il puise dans sa propre biographie. Par exemple, l'amour ne sera jamais traité de façon conceptuelle, mais une touche personnelle et anecdotique apportera un éclairage subjectif et sensible sur cet état affectif. Lanecdote a ainsi pour fonction d'illustrer; elle propose un exemple concret mémorisable d'une réalité plus vaste. En outre, la situation de la scène dans l'espace et le temps (par une phrase introductive ou bien un refrain) permet une forte localisation de ces chansons. C'est en ce sens que nous pouvons qualifier le contenu des ballads $\mathrm{d}$ ' " incidents biographiques locaux ». N'oublions pas, cependant, que ces ballads seraient incomplètes sans les airs qui les accompagnent. Chantées, la plupart du temps, a capella, c'est la voix qui matérialise l'union de la musique et des mots. M. J. C. Hodgart (1950 : 46) décrit cette unité ainsi :

"They cannot achieve their full effect unless they are sung to their own particular tunes $[\ldots]$. They are relatively thin in poetic texture, but their musical setting adds considerably to their richness and profundity [...]. The beauty of the music transforms those words into a more intense expression of feelings $"$ ?.

Les airs sont également des supports efficaces de la mémoire; ils sont faciles à retenir (l'air est répété tout au long de la chanson) et amènent presque naturellement les mots à la bouche. De plus, on compte davantage de textes que d'airs (qui comprennent un certain nombre de variantes à un seul et même air), ces derniers étant souvent connus sous forme instrumentale depuis plus longtemps, la mémorisation des récits est ainsi facilitée.

Revenons à Molly Malone. Cockles And Mussels répond, en tous points, aux critères de la ballad:

- Le récit est contextualisé dans l'espace grâce à ses premiers vers, mais également dans la suite du texte. Il est ancré dans un lieu précis en un instant donné, la scène est visuellement perceptible par l'imagination du lecteur-auditeur.

- L'origine de la narration est une anecdote qui peut se résumer ainsi : un homme croise le regard de Molly Malone qui, peu de temps après, meurt, après avoir attrapé la fièvre: "I first set my eyes on sweet Molly Malone». L'histoire est subjectivée, elle est celle d'un homme qui regarde une femme, et répond en ce sens à la notion d'incident biographique.

9. «Elles ne peuvent réaliser leur plein effet sans être chantées sur leur propre air [...]. Leur texture poétique est relativement pauvre, mais leur arrangement musical ajoute considérablement à leur richesse et leur profondeur [...]. La beauté de la musique transforme ces mots en une expression émotionnelle plus intense» (ma traduction). 
- Le refrain rythme l'ensemble du texte en reprenant la formule de l'air. La chanson est alors facilement mémorisable grâce à ce couplet. La sonorité mélodique des sons utilisés, presque onomatopéiques, garantit la musicalité de la chanson tout en y ajoutant la possibilité d'en saisir l'émotion.

\section{Le contexte d'énonciation}

Intéressons-nous maintenant au contexte d'énonciation du texte ou plutôt à l'événement de chanter. Afin de mieux comprendre cette pratique, je prendrai comme exemple la pratique du chant informelle, celle qui se déroule au pub, tard dans la nuit, entre amis ou en famille.

Les ballads font l'objet d'une pratique en quelque sorte permanente dans chacune des régions d'Irlande. En effet, on chante en de nombreuses occasions, qu'elles soient officielles (mariage, anniversaire, Noël, Jour de l'an, Pâques, etc.) ou bien officieuses (le plaisir de rencontrer ses amis ou sa famille au pub). Ces occasions ont pour point commun le plaisir d'être ensemble, et c'est bien de là que viendra le désir de chanter. Chanter seul n'a pas de sens, c'est avant tout une pratique collective. Toutefois, on ne se retrouve pas au pub pour chanter mais bien plutôt pour passer un bon moment ensemble et discuter. Et c'est justement lorsqu'il y aura eu une "bonne» conversation, des échanges agréables, que naitra l'envie de partager un chant et de se faire entendre. Entretenir des liens forts se pose donc comme condition de l'acte de chanter. Le lieu où l'on chante reflète la socialité de cet instant. Le pub est, en effet, l'espace social privilégié du village - lieu de partage et de conversations. Et le chant s'inscrit parmi ces activités. Nous comprenons alors mieux l'absence de chanteurs professionnels, d'espace scénique et d'amplification, le cour de l'action étant le groupe assis autour d'une table qui partage un bon moment de paroles et de rires. Chacun peut, à tout instant, faire entendre sa voix, être le chanteur du moment. C'est à un tour de table auquel nous assistons, chacun prenant le sien à la suite du précédent. Malgré l'aspect informel que je viens de décrire, il n'en demeure pas moins évident qu'un nombre de règles, implicites mais inviolables, ordonnent le moment de chant.

Pour n'en citer que quelques-unes :

- L'impossibilité de monopoliser le temps de parole, un chanteur ne peut chanter seul toute la soirée.

- On ne peut refuser de chanter lorsqu'une demande est adressée; au mieux, peut-on se faire attendre.

- L'obligation de respect envers le chant d'autrui : le silence ne doit être brisé sous aucun prétexte, il faut écouter. 
- L’obligation de chanter sérieusement. J'entends par "sérieusement", l'impossibilité de chanter "pour de faux "; le chanteur doit s'appliquer et s'investir. Cette condition ne s'oppose pas à la festivité du moment.

Le non-respect d'une de ces consignes rompra l'ambiance intimiste qui s'est établie, le moment de chant sera interrompu. Ces règles nous renseignent finalement sur les valeurs en vigueur : le respect et l'écoute de chacun envers chacun mais également envers le chant. En effet, ce n'est pas tant le chanteur que l'on acclame et respecte que le chant. Ce constat se confirme au vu de la posture propre qui est alors adoptée. Chacun ferme les yeux, il n'est plus question de rire ou de bavarder mais bien plutôt de ressentir, de se concentrer sur l'émotion que génère le chant. Il en va de même en ce qui concerne le chanteur : lui aussi ferme les yeux, son corps est immobile, se faisant véhicule d'une parole chantée. C'est ainsi que l'observateur peu averti pourra avoir quelques difficultés à savoir d'où le chant émane. Celui qui est applaudi est alors «l'un de nous " (plus qu'un chanteur) qui a interprété une chanson connue de tous. Chacun est ici le public de tous, et il est nécessaire au groupe pour qu'il y ait chant. Le chant n'existe donc qu'en présence de tous; il est le fruit d'une interaction, plus que d'une technique vocale; il est, comme le note Bernard LortatJacob (1998: 30), le "témoignage acoustique de présences partagées ".

Cette interaction se situe au cœur de la vie sociale de village en faisant participer chacun à une activité collective. Elle comporte une dimension cérémonielle, au sens durkheimien du terme ${ }^{10}$ de par les deux attitudes qui s'en dégagent: la solennité et la célébration. La solennité de cette interaction est le fait des règles silencieuses qui ordonnent le moment $\mathrm{du}$ chant, mais également du grand respect et du sérieux qui lui sont accordé. Les participants sont donc contraints implicitement à un certain comportement, et ce sont ces règles qui permettent qu'il y ait chant. Néanmoins, nous ne saurions oublier que les cris de contentement, les effusions et les conversations à tue-tête (entre les chants) dénotent un aspect festif relevant du plaisir d'être ensemble. Le moment du chant, tout en étant implanté dans la sphère de la vie quotidienne, s'en démarque en s'imposant comme l'acmé du groupe. C'est donc bien à une célébration à laquelle nous assistons, célébration de l'être-ensemble d'un groupe.

Chanter ensemble peut dès lors être une action sans cesse répétée, il ne pourra y avoir de lassitude puisque ce sont les liens entre les individus que l'on pratique et que l'on écoute. Ainsi, un chant ne sera jamais le même aux yeux des participants, il dépendra des relations que l'on entretient.

10. Tel que l'auteur l'a développé en 1912 dans Les Formes élémentaires de la vie religieuse (cf. Durkheim 1991 [1912]). 


\section{Chanteur et interprétation}

Revenons maintenant à l'énonciation même, à la manière dont le chanteur traite le texte. Les ballads se chantent a capella, avons-nous dit, ou bien accompagnées d'un instrument se contentant de suivre la voix, qui est donc l'instrument principal de cette pratique musicale. Le chanteur est, avant tout, un interprète. L'espace d'un instant, il se fait le porte-voix d'une chanson déjà connue de tous. Nous remarquerons d'ailleurs que les chansons, qui ne sont pas connues d'avance par les auditeurs, ne connaissent pas le même succès ni le même respect; l'écoute est moins attentive et le contentement moins grand.

Mais qu'est-ce qu'une interprétation ? Au sens littéral, une interprétation est avant tout l'effort de rendre audible, de donner corps et sens. Le chanteur doit alors par son interprétation donner un sens au texte de la chanson choisie, le rendre compréhensible, lui donner du corps par la voix. Afin de comprendre comment le chanteur interprète une chanson, intéressonsnous à celui qui est défini comme "bon chanteur " par le groupe. Le "bon chanteur " est généralement un vieil homme (beaucoup plus rarement une femme). Le "bon chanteur" n'est pas celui qui chante juste. Il est celui qui donnera le plus d'émotion, non seulement à ses auditeurs, mais également à lui-même. En ce sens, un chanteur finissant son chant au bord des larmes sera plus applaudi que celui qui se contentera de chanter juste. C'est donc la justesse de l'émotion qui est valorisée, non point tant celle de la musique. Comme nous l'avons vu, dans les ballads, l'émotion n'est pas au cour de la narration. Il s'agit dès lors pour le chanteur de ressentir l'histoire, de la vivre par le chant pour y insuffler ses propres émotions. C'est par sa propre expérience $d u$ vécu que le chanteur va ajouter cette dimension au récit. Dans un entretien, Joe Heaney, chanteur de renom, décrit bien ce processus :

E.W. - "How did you know, when you were singing something, if it's wasn't good?" J. H. — "Sure, I judge it by the way I feel. Now, do I feel this or don't I ? That's the question I ask myself all the time. Do I feel this song, do I put myself in the man's name that this particular song was written about. Am I suffering the labours he did, can I go through that or have that picture before me? if I can't follow that man, the journey he took, whether he was in bondage or slavery, I don't follow the song and I don't do it justice, and I know I don't if I don't do that $"{ }^{11}$.

11. Extrait de l'interview tenue par Esther Warkov in Coleman (1997: 35).

(E.W. - "Comment sais-tu, quand tu chantes quelque chose, si ce n'est pas bien?"

J. H. - «C'est sûr, je le juge par ce que je ressens. En cet instant, est-ce que je ressens quelque chose ou non? C'est la question que je me pose tout le temps. Est-ce que je ressens cette chanson, est-ce que je me suis mis dans la peau de cet homme, dont l'histoire est écrite dans cette chanson. Est-ce que j'éprouve les peines qu'il endure, est-ce que je peux passer par là ou bien avoir cette image devant moi? Si je ne peux pas suivre cet homme, le chemin qu'il a emprunté, qu'il fut dans l'asservissement ou bien même l'esclavage, je ne suis pas la chanson et je ne lui rends pas justice, et je sais que ce n'est pas le cas quand je ne fais pas ça "; ma traduction). 
Il explique, dans la suite de l'entretien, que pour obtenir ce sentiment, il faut vivre la chanson afin d'élaborer un climat imprégnant aussi bien le chanteur que son auditoire. C'est un procédé d'empathie qui est employé ici, permettant au chanteur de rejoindre son texte. Joe Heaney approfondit l'analyse de sa technique en déclarant que l'impératif pour bien chanter est de playing the act, "jouer le jeu, jouer la scène ". Le chanteur va alors piocher dans sa propre expérience pour rendre l'histoire audible, lui donner un sens. Nous sommes dans la définition même du mot "interprétation ». Toutefois, l'expression employée par Joe Heaney nous mène vers une vision théâtrale de l'interprétation, plus proche de la notion de « représentation » et de drame. Donner corps à l'histoire par la voix est, en ce sens, à comprendre littéralement; il ne s'agirait plus seulement de rendre audible un texte mais bien de le rendre visible.

Nous comprenons mieux pourquoi il est impératif de chanter sérieusement, on ne peut se divertir des sentiments personnels. C'est aussi une explication au fait que les personnes âgées connaissent davantage de reconnaissance, elles ont pour elles un vécu théoriquement plus vaste et une expérience plus grande à leur disposition.

\section{Les techniques du "rendre visible"}

Posture et technique vocale

Comme je l'ai précisé précédemment, le chanteur n'est pas remarquable de par sa posture. Il se tient assis comme les autres, rien ne vient le mettre en avant, ni geste ni rythme du corps. Son attitude pourrait plutôt être qualifiée de repli sur soi : yeux clos, mains ramenées sur les genoux, corps en retrait. On voit alors un homme en position de parler de soi, de se livrer intimement. L'écoute des autres et les encouragements, dont il est le sujet, renforcent cette impression. Il en va de même pour les accolades qui clôtureront le chant tels des gestes de soutiens amicaux. Il n'est pas question, ici, de faire de la musique, mais de donner quelque chose de soi, de partager. D'ailleurs, les moments du chant ne seront jamais qualifiés dans le langage de ses protagonistes comme "musique», mais seront désignés par le terme de craic: "passer un bon moment ensemble».

Le chant commence et le silence règne. Le chanteur utilise alors des ornementations pour personnaliser cette interprétation. Les notes importantes peuvent ainsi être allongées et sont généralement en rapport avec les mots que le chanteur souhaite accentuer. Lornementation permet de donner à l'air un aspect émotionnel, de rendre certaines notes plus expressives. L'accent est de la sorte personnel. Un «bon chanteur» utilisera l'alliance des mots et de la musicalité pour donner sa propre signification de la 
chanson. Par ce procédé, l'histoire devient manifeste dans la forme mélodique même, au-delà du texte. L'anecdote narrée dans la ballad n'est donc pas directement mise en scène par le chanteur; il ne la reproduit pas; il joue plutôt le rôle du narrateur. La technique utilisée par le chanteur, décrite par Joe Heaney, de vivre le chant est rendue visible et audible, sa posture tendant à confondre celui qui chante et celui à qui l'histoire est arrivée : le protagoniste. Il s'agit dès lors, pour le chanteur, que l'histoire qu'il est en train de raconter puisse être perçue comme "possiblement réelle » par les auditeurs. Et pour cela, il leur faut la possibilité de l'imager.

\section{Confondre les contextes pour imager}

Le texte supporte de nombreux outils que le chanteur peut utiliser. L'emploi de termes indexicaux est récurrent dans les textes de ballads. Nous en citerons deux en ce qui concerne Cockles And Mussels. Premièrement, l'emploi de la première personne du singulier, renforçant l'aspect biographique de l'anecdote: "I first set my eyes on sweet Molly Malone». Par le «je», le narrateur et le protagoniste se confondent. Deuxièmement, l'utilisation de now, "maintenant " dans : Now her ghost wheels her barrow, introduit une conjugaison au présent du verbe. En effet, now a également cette particularité de pouvoir se référer au contexte d'énonciation présent tout autant qu'au contexte de l'histoire passée. Une intertextualité est entretenue par un principe de citation directe, mettant en relation le texte et le contexte de l'anecdote. L'aspect vécu du texte est ainsi souligné, la citation apparaît comme un souvenir. Le narrateur semble rapporter ce qu'il a entendu mot pour mot, il rend présent une partie de l'événement passé. L'auditeur est projeté dans la scène. Dans Cockles And Mussels, les paroles de Molly Malone sont rapportées par citation comme suit: "Cockles and mussels, alive alive oh!"

Par ces procédés, le chanteur et le narrateur de l'anecdote deviennent une seule et même personne, l'événement passé est ramené dans le présent. Ainsi, l'anecdote n'est pas située historiquement dans le temps, elle est plutôt de l'ordre de l'atemporalité. La contextualisation topographique est également une spécificité de nombre de chants. Dès les premiers vers de Cockles And Mussels, l'auditeur sait où se déroule la scène, il peut donc la situer et lui donner un arrière-plan, imaginer un paysage. Chaque chanson est locale par son adaptation à la topographie du lieu où elle est chantée. Nous observons parmi le répertoire irlandais un certain nombre de formules archétypales utilisées pour les titres, et modifiables à souhait. C'est le cas par exemple de The Lass Of Aughrim que l'on retrouve décliné ainsi: The Lass Of Ormond, The Lass Of Arram, etc. Le chant est, par spatialisation du récit, toujours possiblement imageable par ceux qui 
écoutent et pour celui qui chante. Chaque membre de la localité peut dès lors se reconnaître dans la chanson, l'identifier comme étant celle de son groupe, voire de son village, de son quartier ou de sa ville. Cockles And Mussels avait alors un public tout trouvé à Dublin, grâce à ses deux premiers vers.

\section{Une biographie fictionnelle}

Les procédés décrits plus haut visent en tous points à mélanger les contextes. En effet, le chanteur devient le protagoniste de l'anecdote : cette histoire devient la sienne, ce sont ses propres émotions que l'on entend. En outre, la scène de l'histoire est localisable dans l'espace, elle s'est déroulée «ici» même. C'est ainsi que le chanteur expose, montre son histoire ; l'événement passé est ranimé dans le présent par un effet de spatialisation, renforcé par l'indexicalité. Cet enchevêtrement des contextes est rendue possible par l'intimité dans laquelle se déroule le chant; en effet, n'oublions pas que ceux qui sont présents partagent des références communes en raison des liens forts qu'ils entretiennent entre eux. Ainsi, chaque élément de la chanson pourra être référé par le groupe à une réalité. L'interprétation n'est donc pas seulement due au chanteur mais est également une visée collective de la part de ceux qui écoutent son chant, une tentative pour ressentir ces paroles, les comprendre dans ce contexte. Ainsi, le chanteur, en rendant audible un texte, le pare également d'un sens commun, celui du groupe. L'histoire racontée par Cockles And Mussels n'est plus seulement celle de Molly Malone mais celle de cet homme connu de tous qui nous dit quelque chose de lui. C'est l'interprétation du "je» qui est jugée par l'écoute des auditeurs.

Nous pourrions alors dire qu'en jouant la scène de raconter l'anecdote, le chanteur incarne l'histoire en se l'appropriant, il en fait une part de son expérience que, au travers de son corps, il donne à voir à ceux qui sont présents. Le chant devient un processus collectif de visualisation et de matérialisation dans l'espace qui donne vie aux anecdotes, leur confere une réalité. Finalement peu importe, pour les auditeurs, la vérité de l'histoire face à la réalité des sentiments exprimés et partagés en cet instant. Il ne s'agit pas de dire que le groupe tient ces anecdotes pour vraies mais que la réalité de l'histoire est envisagée, par chacun, par l'identification possible à l'expérience sensible qu'elle relate.

Par la pratique collective du chant et par l'émotion qu'il communique, ce sont donc des expériences personnelles qui sont mises en partage. En les donnant à voir, le chanteur les transmet. Et ce rôle du chanteur peut être rapproché de celui du narrateur tel que l'a décrit Walter Benjamin (1991 [1969] : 87) : 
"The storyteller takes what he tells from experience - his own or that reported by others. And he in turn makes it the experience of those who are listening to his tale " ${ }^{12}$.

La répétition des chants est alors compréhensible, elle permet à chacun de créer ses propres intentions de signification. L'adaptation du texte à des significations locales relève d'un principe de fiction, l'appropriation s'effectuant par un jeu d'identification. L'anecdotique constitue un procédé narratif relevant de la biographie que je qualifierai de fictionnelle en tant qu'elle est ancrée dans la réalité sociale et l'imaginaire symbolique d'un groupe et non dans sa vérité historique.

\section{De la biographie fictionnelle à la vérité historique}

Au regard de la pratique du chant par un groupe, nous comprenons l'indissociabilité du texte et de son contexte, du texte et de sa pratique. C'est l'interprétation, d'une part du chanteur et d'autre part des auditeurs, qui matérialise l'anecdote, qui lui donne un sens. En parlant ici de contexte du texte, nous prenons en compte les deux dimensions de celuici: l'événement chanté et l'événement qu'est le chanter. En effet, le mélange du contexte d'énonciation et du contexte dans lequel l'histoire se déroule comme technique narrative nous amène à les fondre en un seul mot. C'est par cette unité indissoluble du texte, de l'acte de chanter et de l'événement chanté que Richard Bauman (1999: 7) définit la performance orale. Cette unité est le fruit d'une pratique interactionnelle, éminemment sociale, par laquelle le performer soumet son chant à un auditoire pouvant le valider ou bien l'invalider. Le chant est ainsi compris dans un contexte local de connaissances et de références ${ }^{13}$.

Le terme de "performance» est d'autant plus pertinent ici que sa traduction française sous la notion de "représentation", dans l'œuvre de Goffman, met en lumière un des aspects de celle-ci. En effet, cette traduction exprime l'idée d'une mise en scène telle que nous avons pu la constater dans le chant: le performer représente une chanson en lui donnant corps.

12. «Le conteur prend ce qu'il raconte de l'expérience - la sienne ou bien celle rapportée par d'autres. Et, par un tour, il la fait devenir l'expérience de ceux qui écoutent son histoire» (ma traduction).

13. "Oral performance, like all human activity, is situated, its form, meaning, and functions rooted in culturally defined scenes or events - bounded segments of the flow of behaviour and experience that constitute meaningful contexts for action, interpretation, and evaluation " (Bauman 1999 : 3). («La performance orale, comme toute activité humaine, est située : sa forme, sa signification et ses fonctions sont enracinées dans des scènes ou événements culturellement définis - les cadres du flux de comportements et d'expériences constituent des contextes signifiant pour l'action, l'interprétation, et l'évaluation"; ma traduction). 
Pour revenir à nos interrogations premières, nous pouvons tenter de répondre à la question de savoir si Cockles And Mussels est bien un « objet de tradition ». Dans le cadre de la performance, cette chanson répond aux critères de sélection qui lui avaient fait défaut lors de son évaluation dublinoise. En effet, Cockles And Mussels telle que nous l'avons décrite dans sa pratique est profondément locale en tant qu'elle se matérialise dans un espace partagé par ceux qui la chantent. Ce n'est donc pas le texte original qui détermine l'appartenance d'une chanson à un lieu, mais un processus collectif d'appropriation l'enracinant dans une localité. C'est la circulation orale qui en fait un objet local. Ce processus fait des chansons des objets atemporels. Elles ne connaissent pas de datation historique puisqu'elles existent de nouveau à chaque reprise, à chaque chant. Pour ceux qui les pratiquent peu leur en importe l'origine, c'est la continuité qui accorde une valeur à ce qui est chanté. Ainsi, pour un chanteur, savoir que sa mère chantait la chanson qu'il chante lui-même est une garantie d' " authenticité " au-delà d'une date originelle de création. De plus, une chanson n'est jamais complètement nouvelle, comme nous l'avons vu; elle conserve toujours un certain nombre de références à quelque chose de connu auparavant. Une chanson ne peut donc être déclarée comme trop récente par ceux qui chantent, à partir du moment où elle s'inscrit dans une logique de continuité.

Parlons maintenant du critère d'anonymat validant une chanson traditionnelle. Dans la pratique que nous avons décrite, l'auteur des textes n'est pas vecteur d'intérêt, c'est l'interprète qui est mis en lumière. Le performer ne se réduit pas ici à un rôle passif de "conducteur" anonyme de la tradition collective (Bauman 1999: 8) ${ }^{14}$. L'interprétation qu'il fait du texte en prenant la parole est toute personnelle, il donne à la chanson son propre sens. Cette "innovation" sera soumise à l'acceptation du groupe qui l'écoute. Il est donc nécessaire de distinguer création et re-création. La re-création peut être collective et orale alors que la création du texte peut être l'œuvre écrite d'un individu isolé. Le but de la pratique collective du chant est, dans notre cas, une répétition créatrice de sens et non une création absolue de texte. Il s'agit de valider les connaissances du groupe et de les transmettre. La chanson n'appartient donc à personne en particulier, elle n'existe que lorsqu'elle est pratiquée et de la sorte appropriée, d'où la nécessité de chanter pour la transmettre et se l'approprier. La présence d'un auteur n'est, dans cette pratique, absolument pas problématique. Plusieurs auteurs, pour la plupart poètes, sont désormais reconnus pour

14. Richard Bauman décrit le risque de considérer le performer ainsi lorsque l'objet d'étude se porte sur les formes d'expressions collectives. 
leur travail d'écriture. Les textes, ainsi écrits, ne sont pas mis à l'écart dans la pratique quotidienne que les Irlandais peuvent avoir des chants, ils suscitent le même intérêt que les textes anonymes. Ainsi pouvons-nous citer Percy French (1854-1920), auteur notamment de The Mountains Of Mourne, Thomas Moore, auteur de The Minstrel Boy, Peadar Kearney qui, outre l'hymne irlandais, a écrit Down By The Liffey Side, et bien d'autres encore. Le même constat s'est établi après quelques entretiens que j'ai pu avoir avec des chanteurs de village (non professionnalisés), à la question "comment as-tu appris cette chanson?", il n'était pas incongru de répondre "grâce à mon livre de chansons ". Peut-être est-ce la notion d' " oralité seconde» proposée par Walter Ong (1982) qui serait le mieux à même de nous approcher de la réalité en écartant la dualité de l'écrit et de l'oral, de l'individuel et du collectif. L'écrit serait simplement un média de la communication orale, un support à la remodélisation collective. En ce sens, un texte peut pénétrer la tradition orale jusqu'à ce qu'on en oublie son origine, à force de circulation, à force d'appartenances successives à des groupes. C'est également le constat de Sean Murphy (1992: 7) quant à Cockles And Mussels :

«It must have gained such popularity and been so widely dispersed that its origins were lost to memory and it was assumed to be just another anonymous folk song ${ }^{15}$.

Le chant est donc profondément oral et anonyme alors que la chanson peut appartenir au domaine de l'écrit et avoir un auteur, et ce, sans qu'il y ait contradiction.

L'objet de tradition n'existe ici que dans son contexte d'énonciation, ce n'est donc pas l'objet qui est traditionnel mais la pratique que l'on en a et que l'on en fait. Davantage que la chanson, c'est le chant en tant que pratique qui est une tradition. La performance «chant» rend son objet «chanson" anonyme, atemporel et local.

\section{Pendant ce temps, à Dublin...}

Ce n'est qu’à partir des années 1960 que Dublin connut un développement économique. Ce développement urbain fut aussi celui du secteur culturel avec l'apparition des temps de loisirs. La musique et donc les ballads suivirent ce mouvement d'urbanisation et de consommation de masse. Mais c'est aussi l'époque où les immigrés irlandais aux États-Unis

15. «Elle a gagné tant de popularité et a été si largement dispersée que son origine a été perdue dans les mémoires et il fut supposé qu'elle était juste une autre chanson populaire anonyme » (ma traduction). 
percèrent dans le milieu musical, les compagnies américaines cherchant de nouveaux talents. Ce phénomène fut amplifié par le mouvement de retour aux sources qui marqua la tradition musicale américaine dans les années 1960-1970. Cet engouement nouveau pour la musique irlandaise contribua lentement à effacer son image rurale et arriérée; c'était désormais un genre dans lequel il était possible de réussir et de se faire entendre de par le monde. C'est donc sous forme d'enregistrements que la musique irlandaise "revint " à Dublin, finalisant l'urbanisation musicale en cours. Une autre pratique musicale émergea, parallèlement à la pratique « informelle » se déroulant au pub, caractérisée par une professionnalisation de ses musiciens. Des groupes se constituèrent, reprenant les chansons dites «traditionnelles " à la mode urbaine. Le contexte d'énonciation des chants en fut considérablement modifié : les groupes se produisent sur scène, devant un public, avec une amplification de la voix et des instruments. Le chant n'est plus une parole que l'on écoute mais une musique que l'on vient entendre pour se divertir. Dans ce cas de figure, la dimension esthétique (et technologique) du chant supplante l'aspect d'expression intime et collective de communication villageoise, que nous avons décrit précédemment. Le chant est ici un art, et trouve sa place comme genre musical sur les scènes internationales.

Ainsi classée au rang d'art, la musique dite traditionnelle devint l'objet de réflexivité de la part de ses nouveaux adeptes. Le besoin se fit sentir de l'étudier, de la comprendre pour mieux la protéger et la faire vivre. En effet, cette musique qui se pratiquait sur les scènes dublinoises arrivait tout droit des États-Unis; il fallait désormais regarder chez soi afin de trouver de nouveaux matériaux. Cette prise de conscience, d'un héritage riche, amorça la nécessité de définition de ce nouvel objet.

C'est dans ce contexte qu'émergèrent les questionnements décrits par Sean Murphy au sujet de Cockles And Mussels. Si cette chanson devait intégrer le patrimoine irlandais, non seulement au niveau national mais international, on se devait d'en connaître précisément l'origine. C'est à ce moment qu'apparurent les critères de sélection cités précédemment: la date, le lieu, l'anonymat. En effet, la patrimonialisation exige de la tradition, qu'elle soit donc là «depuis toujours » en ce lieu. L'aspect traditionnel de Cockles And Mussels ne pouvant être démontré par le texte, il devait être prouvé par la pratique. En regardant de plus près comment on pratiquait cette chanson "depuis toujours", les protagonistes de ces débats espérèrent dénicher une preuve de l'existence de Molly Malone.

L'urbanisation des ballads eut pour première conséquence une décontextualisation du texte. Cockles And Mussels pouvait désormais exister sans la participation de tous et de chacun, grâce aux enregistrements. Le chant 
n'est plus ici une production collective, le fruit d'une interaction, mais un produit fini que l'on peut réécouter à souhait. Une attention toute particulière se porte alors sur le texte, sur le message, sur ce qui est dit dans la chanson, au détriment de l'intérêt pour l'histoire personnelle de ceux qui la chantent. Or, l'interprétation du chant n'est plus aux confins de l'anecdote, de l'histoire personnelle du chanteur et de l'histoire du groupe qui l'écoute. Par cette mise en exergue de l'événement narré, Molly Malone emprunta le premier rôle au " je " peu audible du troisième vers, et surtout peu «visible» à l'écoute d'un enregistrement. En effet, pour que le «je » ait un impact sur l'auditeur, il fallait que l'identification avec le chanteur fût visible, c'est-à-dire qu'il lui fallait entretenir des liens forts avec cet homme pour comprendre le sens de son interprétation. De la sorte, ce n'était plus l'histoire d'un homme parmi tant d'autres mais bien celle de Molly Malone. Ce constat se confirma au vu de la modification progressive du titre de la chanson dans les banques de données, nous la trouvons couramment sous le titre de "Molly Malone». L'accent n'est plus mis sur l'aspect anecdotique du refrain Cockles And Mussels mais bien sur son personnage principal. Ce n'est donc plus le chant d'un homme que l'on écoute mais l'histoire de Molly Malone, ce qui entraîne un déplacement de signification.

Tous les procédés narratifs décrits peuvent, en effet, donner à Cockles And Mussels un sens nouveau si l'on se concentre sur son personnage principal. Comme nous l'avons dit, ces techniques visent à rendre la chanson vivante, à l'incarner dans son chanteur. Si l'histoire se recentre sur Molly Malone, alors, ce n'est plus seulement l'identification du chanteur avec le protagoniste de l'anecdote qui est susceptible d'être réelle mais le personnage lui-même. En effet, si le chanteur est un "bon chanteur", tel que nous l'avons décrit, il incarne Molly Malone en la faisant vivre par son chant, lui donne un visage et un paysage au travers de la réalité de ses émotions.

L'«incident biographique» décrit par l'anecdote renforce cette possibilité de penser le personnage comme ayant existé. Ces petites histoires n’ont rien de fictives, elles sont bien ancrées dans la vie quotidienne; c'est la posture envers elles qui peut être fictive. Or, le recentrement sur le personnage de l'histoire modifie cette posture, ce ne sont plus les émotions partagées qui sont réelles mais Molly Malone. Ainsi, soit le « je » est réel et Molly Malone est un incident biographique fictionnel, soit Molly Malone est réelle et le " je » chante sa propre biographie. Comme nous l'avons dit, la chanson s'inscrit dans une réalité partagée d'un groupe. Déracinée de ce contexte, Molly Malone devient une vérité historique, et il n’y a plus de posture fictionnelle devant la réalité des faits. 
La dimension cérémonielle du chant tend également en ce sens : le respect et la solennité exprimées en ce moment peuvent être interprétés comme une croyance partagée en la réalité de l'histoire. Le sérieux, lisible sur les visages, démontre que l'on ne peut rire avec les chansons, tels des secrets que l'on livre. Par ce décentrement d'attention, c'est également l'aspect biographique qui est mis en avant, à une petite différence près : ce n'est plus celui du chanteur mais bien celui de Molly Malone. La biographie revêt alors une nouvelle dimension, elle n'est plus une identification par l'expérience au texte de la chanson, mais une historicisation de l'anecdote. En effet, lorsque Cockles And Mussels n'est plus imagée dans l'espace par les références partagées, s'opère un arrêt sur image cristallisant Molly Malone dans une scène localisée dans le temps. Son décor ne varie plus à chaque chant, selon chaque groupe, mais se fige dans le passé. La statue, dressée en 1989, symbolise cette nouvelle posture urbaine quant aux chants. Les recherches historiques qui eurent lieu, afin d' « habiller» Molly Malone (dans sa sculpture), confirme cette volonté de la replacer précisément dans le temps. Plus qu'une image partagée par un groupe, créée par une interaction, Molly Malone est désormais représentée matériellement par une sculpture que l'on peut aller voir. Le «rendre visible» du chant n'est plus une abstraction mais une réalité concrète.

En définitive, la décontextualisation du texte se caractérise par l'absence d'association du contexte d'énonciation et du contexte de l'événement chanté. La vie de Molly Malone est inexorablement un événement appartenant à l'histoire. Cette historicité acquise, Molly Malone peut, non seulement avoir existé, mais être une vérité historique. Tenir une histoire pour vraie est le propre d'un processus mythifiant. Et ce processus s'incarne concrètement par les recherches engagées pour retrouver Molly Malone. Lui donner une date de naissance et de mort ainsi qu'une sépulture, puis une sculpture, achève l'historicisation de cet incident biographique.

\section{Une héroïsation réussie}

Pour que ce processus mythifiant s'opère, il fallait une population dublinoise prête à cela. Il fallait que Molly Malone pût représenter, en quelque sorte, une héroïne pour la ville, qu'elle symbolisât un sentiment collectif. Pour expliquer l'héroïsation réussie de celle-ci, il convient de prendre en compte l'histoire de l'Irlande. En effet, l'occupation, voire l'oppression anglaise, dura bon nombre de décennies. L'Irlande n'obtint son indépendante totale qu'en 1937. L'histoire de ce pays est marquée par de nombreux drames, dont la "grande famine " à cause de laquelle l'Irlande perdit une partie de sa population. Ces souffrances ont été exacerbées par 
l'emprise anglaise sur l'histoire irlandaise. C'est ainsi, encore aujourd'hui, qu'un certain nombre d'événements historiques conservent leur version anglaise des faits et font débats. Pour reprendre un exemple bien connu (sans oublier qu'il y en a bien d'autres) - celui de la "grande famine " l'implication anglaise dans la mort de millions d'Irlandais n'est pas encore officiellement reconnue. Démunis de tous moyens d'expressions, il ne restait aux Irlandais que leur musique. Celle-ci fut également censurée voire interdite par les protestants, la considérant comme dangereuse à l'établissement de leur pouvoir. Toutefois, la musique et les chants continuèrent à se faire entendre officieusement là où les autorités ne pouvaient les saisir. Comme l'explique Mick Moloney (2002) dans son ouvrage, seule la tradition orale permettait l'unité culturelle et historique des Irlandais. C'est dans ce contexte qu'il faut replacer Cockles And Mussels, l'anecdotique illustrant ici comme dans les autres ballads une réalité vécue. En effet, Molly Malone meurt, dans le texte, d'une fièvre. Celle-ci peut avoir plusieurs origines mais symbolise, dans tous les cas, une souffrance et des conditions de vie précaires. Le sentiment collectif d'injustice face à l'histoire trouve en cette chanson un cri, une affirmation, d'un vécu peu reconnu. Cockles And Mussels, en incarnant ce sentiment, devient un symbole de l'identité dublinoise et Molly Malone une héroïne de l'histoire, que l'on peut exhiber aux "autres" pour qu'ils entendent, pour qu'ils voient. Ce n'est donc pas l'esprit de la ville qui vit au travers de cette chanson mais bien un sentiment collectif d'individus ayant partagé une histoire. Les débats résumés dans le début de cet article ont donné à Molly Malone un parcours atypique. En effet, la volonté de la déclasser en musique populaire tout aussi bien que de la restituer à son auteur écossais a profondément choqué les Irlandais, comme si une partie de leur histoire leur avait été dérobée. La chanter est désormais une réaffirmation de l'identité dublinoise face à ceux qui ont voulu la rendre (ou la laisser) aux Écossais. Nous comprenons alors que Cockles And Mussels soit aujourd'hui l'hymne officieux de la ville de Dublin.

Le processus mythifiant mis à l'œuvre ici n'est pas seulement une rumeur urbaine mais s'inscrit au cœur d'enjeux politiques et identitaires. Ce n'est donc pas tant la décontextualisation urbaine de la chanson que ces enjeux qui ont propulsé Molly Malone en haut de l'affiche. Finalement, nous pouvons supposer que l'urbanisation de la pratique des chants n'a pas modifié fondamentalement les principes même qui la régissent. La pratique «rurale » des chants a permis un processus mythifiant en proposant un certain nombre d'éléments qui, détachés de leur contexte, 
étaient le vecteur d'une autre signification. Le passage d'une pratique collective du chant officieuse à une pratique scénique officielle a déplacé l'attention de l'auditeur et du chanteur sur le personnage principal de la ballad. Ce n'est plus la pratique du chant qui est au cœur de l'interaction mais son objet : la chanson. Toutefois, la démarche réflexive des artistes et intellectuels dublinois s'inscrit dans un contexte local et historiquement situé. L'interprétation de la chanson n'est pas ici le fait d'un petit groupe entretenant des liens forts mais celle d'une ville politiquement impliquée dans son identité. Il n'y a donc pas décontextualisation du texte en milieu urbain mais recontextualisation et déplacement de signification. Nous ne sommes plus dans une contextualisation spatiale de la ballad mais bien dans une contextualisation historique du texte. Le propre de la pratique du chant serait alors une contextualisation collective permettant une identification d'un groupe à une histoire, permettant de donner une forme aux expériences partagées. Les ballads peuvent ainsi représenter des groupes d'individus - qu'ils soient groupe d'amis, ou famille, village et ville - qui, en chantant, brandissent leur histoire.

Laboratoire d'anthropologie et d'histoire de l'institution de la culture, Ehess, Charenton-le-Pont charlottepoulet@free.fr

MOTS CLÉS/KEYWORDS : ethnographie du chant/ethnography of song - chansons irlandaises/ irish songs - tradition orale/oral tradition - interprétation/interpretation - performance mythification - légende urbaine/urban legend. 


\section{Cockles And Mussels, texte de la chanson tel qu'il a été publié à Londres en 1884 par Francis Brothers \& Day.}

In Dublin's fair city,

where the girls are so pretty,

I first set my eyes on sweet Molly Malone,

As she wheeled her wheel-barrow,

Through streets broad and narrow,

Crying, "Cockles and mussels, alive alive oh!"

"Alive-a-live-oh,

Alive-a-live-oh"

Crying "Cockles and mussels, alive alive oh!".

She was a fishmonger,

And sure 'twas no wonder,

For so were her mother and father before,

And they both wheeled their barrow,

Through streets broad and narrow,

Crying, "Cockles and mussels, alive, alive oh!" (refrain)

She died of a fever,

And no one could save her,

And that was the end of sweet Molly Malone.

Now her ghost wheels her barrow,

Through streets broad and narrow,

Crying, "Cockles and mussels, alive, alive oh!" (refrain)
Dans la belle ville de Dublin

Où les filles sont si jolies

J'ai posé en premier mes yeux sur la douce Molly Malone

Elle poussait sa brouette

À travers les rues larges et étroites

Criant, "des coques et des moules, vivantes, vivantes, oh !"

$$
\text { (refrain) }
$$

Elle vendait son poisson

Sans se faire d'illusions

Ainsi faisaient son père et sa mère

Et ils poussaient leur brouette

À travers les rues larges et étroites

Criant, "des coques et des moules, vivantes, vivantes, oh!"

$$
\text { (refrain) }
$$

Elle mourut d'une fièvre

Et personne ne put la sauver

Et ce fut la fin de la douce Molly Malone

Maintenant son fantôme pousse sa charrette

À travers les rues larges et étroites

Criant, "des coques et des moules, vivantes, vivantes, oh !" (refrain) 


\section{Bauman, Richard}

1999 [1986] Story, Performance and Event. Contextual Studies of Oral Narrative.

Cambridge-New York, Cambridge

University Press.

\section{Benjamin, Walter}

1991 [1969] Illuminations. New York, Schocken Books.

Bradley, John, ed.

1984 Viking Dublin Exposed. The Wood Quay Saga. Dublin, O’Brien Press.

\section{Coleman, Steve}

1997 «Joe Heaney and Style in Sean-

N'os Singing ", in Thérèse Smith \& Micheal Ó Súilleabháin, eds, Blas. The Local Accent in Traditional Irish Music (Selected Proceedings). Dublin, The Folk Music Society of Ireland and the Irish World Music Center, University of Limerick : 33-50.

Durkheim, Émile

1991 [1912] Les Formes élémentaires de la vie religieuse. Le système totémique en Australie. Paris, Librairie générale française («Le Livre de poche»).

\section{Gerould, Gordon Hall}

1974 [1932] The Ballad of Tradition. New York, Gordian Press.

\section{Hodgart, Matthew John Caldwell}

1950 The Ballads. London-New York, Hutchison's University Library.

Lortat-Jacob, Bernard

1998 Chants de passion. Au cour d'une confrérie de Sardaigne. Paris, Le Cerf.

Moloney, Mick

2002 Far from the Shamrock Shore. The Story of Irish-American Immigration through Song. Cork, Collins Press.

Moulden, John

1997 «One Singer, Two Voices : Scots and Irish-Style Song in the Work of the Mid-Antrim Poet and Song Maker Hugh McWilliams (fl. 1816-1831) ", in Thérèse Smith \& Micheal Ó Súilleabháin, eds, Blas. The Local Accent in Traditional Irish Music (Selected Proceedings). Dublin, The Folk Music Society of Ireland and the Irish World Music Center, University of Limerick:

73-99.

\section{Murphy, Sean}

1992 The Mystery of Molly Malone. Dublin, Divelina Publications.

Ong, Walter J.

1982 Orality and Literacy. The Technologizing of the Word. New York, Routledge. 
Charlotte Poulet, Le chant qui donne la vie ou comment Molly Malone est devenue vivante. Un processus mythifiant au cœur de Dublin. Cet article s'intéresse à la chanson irlandaise Cockles And Mussels qui connut un destin particulier. Elle figure, comme bien d'autres, dans les répertoires irlandais de musique traditionnelle tout comme sur les enregistrements présentant le «meilleur de la musique irlandaise ", sans que rien ne la différencie à première vue. Toutefois, elle est devenue l'hymne officieux de Dublin et son personnage principal, Molly Malone, possède désormais une statue à son image dans les rues de Dublin. Plus que le personnage d'une histoire, Molly Malone est devenue une héroïne représentant, aux yeux de tous, sa ville natale. On se propose d'esquisser ici une explication du processus mythifiant qui a été mis à l'œuvre, et cela à partir de l'histoire de cette chanson à Dublin tout autant que par l'ethnographie de la pratique du chant, en Irlande.
Charlotte Poulet, A Life-Giving Song: How Molly Malone Came to Life. A Myth-Making Process in Dublin. - Cockles And Mussels has had a peculiar fate. This song figures on recordings of the "best of Irish music». At first sight, nothing sets it apart from the many other songs in the repertoire of traditional Irish music. Nonetheless, it has become Dublin's unofficial anthem; and a statue to Molly Malone has been erected in the city. More than the major character in the song, Molly Malone has become a heroine representing, for everyone, her native city. The making of a myth is described through the history of this song in Dublin and through an ethnological study of singing in Ireland. 